\title{
On Codebook Design for Distributed Relay Beamforming Network
}

\author{
Min Zheng and Min Dong \\ Department of Electrical and Computer Engineering \\ University of Ontario Institute of Technology, Ontario, Canada \\ Email: \{min.zheng, min.dong\}@uoit.ca
}

\begin{abstract}
We study the problem of codebook design for quantized distributed relay beamforming under individual relay power constraint in a cooperative relay network. The received SNR is used as the design metric. We study two types of codebook design: phase-only codebook where each relay uses full power, and codebook with power control where relay power and phase need to be jointly considered. For the former, we adopt Grassmannian line packing criterion under equal gain constraint, and compare the performance with that under the generalized Lloyd Algorithm for optimal codebook design. For codebook with power control, the generalized Lloyd Algorithm can be used for the optimal codebook design, but incurs high complexity. We propose a low-complexity suboptimal method, which can directly use the optimal power control solution for perfect channel knowledge under the individual power constraint. Through simulation study, it is found that it is more beneficial to use codebook with power control over codebook without power control when relays are closer to destination, instead of the source.
\end{abstract}

\section{INTRODUCTION}

We consider cooperative relaying where several relays assist communications between the source and the destination. When amplify-and-forward (AF) relaying is employed and the channel state information (CSI) is available at each relay, relays can adjust the phase and power of their transmitted signals to form distributed beamforming to maximize received signal-to-noise ratio (SNR) for detection. This allows the system to exploit the benefit of multi-antenna beamforming through distributed relays to enhance the overall performance.

Similar to multi-antenna beamforming, the accuracy of CSI at each relay is critical to achieve full beamforming gain for distributed relay beamforming. Since such information can only be obtained through limited feedback, the main problem is then on how to quantize the channel or beamforming vector under feedback limitation. Even though such problem bears great similarity to that of multi-antenna beamforming in point-to-point systems [1]-[3], the quantization design for distributed relay beamforming has its own unique challenges. This is reflected in the two-hop relaying structure, noise amplification by AF relaying, and distributed nature preventing joint signal processing. In particular, distributed relay beamforming imposes individual power control constraints. This gives rise to unique challenge for quantization design of beamforming vectors not encountered in traditional codebook design. Thus, existing results for multi-antenna systems may not be directly applicable, and quantization design needs careful investigation taking the above factors into consideration.

In this paper, we focus on the problem of codebook design for quantized distributed relay beamforming. Each relay in the network has its individual power constraint. We assume the codebook is known at both relays and destination receiver, and the best beamforming vector is selected from the codebook at the destination and its index is conveyed to the relays. Our codebook design objective is to maximize the received SNR. Due to individual relay power constraint, we consider two types of codebook design: codebook without power control where each relay uses full power, and codebook with power control where relay power adjustment is factored in the codebook design. For the former, the codebook only contains phase information. We adopt Grassmannian line packing criterion [2], [4] to design the phase-only codebook under equal gain constraint, and compare the performance with that under the generalized Lloyd Algorithm [5] for optimal codebook design. For codebook with power control, the generalized Lloyd Algorithm can be again used for optimal codebook design procedure. However, such procedure incurs high complexity in each iteration in searching optimal solution, which is undesirable for online codebook generation. To this end, we propose a suboptimal method, which can directly use the optimal power control solution obtained in [2] for perfect CSI under the individual power constraint. Since the solution is in closed-form, the suboptimal method only requires lowcomplexity computation in generating the codebook. Through simulation study, it is found that it is more beneficial to use codebook with power control over codebook without power control when relays are closer to destination, instead of the source.

Under perfect CSI, the problem of distributed relay beamforming has been studied under both sum-power constraint of relays [6], [7] and individual power constraint [8] for oneway relaying and for two-way relaying [9], [10]. The closedform optimal solution for relay power control to maximize received SNR is obtained under individual power constraint in [8]. It is shown that one or more relays are required to use full power, and the rest use fractional powers to optimally balance the signal vs. noise amplification for relaying. Outage minimization under quantized CSI is studied for single-relay networks in [11]. Under sum-power constraint, the beamforming performance under quantized CSI is studied for AF 
relaying [12]. There is a rich literature on codebook design for multi-antenna point-to-point system under limited feedback [1]-[3]. In [2], Grassmannian line packing criterion was shown to be optimal for codebook design for SNR maximization under i.i.d. Rayleigh fading channels. However, for other various network setups or performance metrics, Grassmannian beamforming is not optimal, and Lloyd's Algorithm [5] has be adopted to generate optimal codebooks [13], [14].

Notation: Throughput the paper, we use $(\cdot)^{T},(\cdot)^{*},(\cdot)^{H}$ to represent the transpose, the complex conjugate, and the Hermitian operators, respectively. $\operatorname{diag}(\mathbf{a})$ indicates a diagonal matrix with diagonal entries given by the elements of vector a, and $\|\cdot\|$ denotes 2-norm. We use $\angle z$ and $|z|$ to denote the phase and amplitude of a complex variable $z$, respectively. $E_{x}\{\cdot\}$ denotes the expectation taken over random variable $x$.

\section{Problem Formulation}

Consider a dual-hop multiple-relay cooperative system, where source node $S$ transmit data to the destination node $D$ through the assistance of $M$ relays, denoted by $R_{m}$, for $m=$ $1,2, \ldots, M$. Each relay is equipped with a single antenna, and it forwards data cooperatively with the rest of relays through distributed relay beamforming. The amplify-and-forward (AF) protocol is used to complete a transmission in two steps. In the first step, node $S$ sends $\sqrt{P_{s}} s$ to $M$ relays, where $P_{s}$ is the transmit power at $S$, and $s$ is the information symbol with $E\left[|s|^{2}\right]=1$. Let $f_{m}$ denote the channel between node $S$ and relay $R_{m}$, and $n_{m}$ denote the AWGN noise at relay $R_{m}$ with $n_{m} \sim \mathcal{C N}(0,1)$. The received signal at relay $R_{m}$ is given by

$$
r_{m}=\sqrt{P_{s}} f_{m} s+n_{m} .
$$

In the second step, all the relays forward the received signal to node $D$. Assume $P_{m}$ is the transmit power constraint on relay $R_{m}$. Under the AF protocol, each relay amplifies the received signal power to $P_{m}$, and multiplies the amplified signal with the conjugate of a beamweight $w_{m}=\alpha_{m} e^{j \theta_{m}}$, where $\theta$ is the phase factor to co-phase forwarded signals among relays, and $\alpha_{m} \in[0,1]$ is the power control factor for the power used at relay $R_{m}$. The transmitted signal at relay $\mathbf{R}_{m}$ is given by

$$
t_{m}=w_{m}^{*} \sqrt{\frac{P_{m}}{1+\left|f_{m}\right|^{2} P_{s}}} r_{m}
$$

Thus, the average transmit power at relay $R_{m}$ is $\alpha_{m}^{2} P_{m}$. Let $g_{m}$ denote the channel between relay $R_{m}$ and node $D$, and $n$ denote the receiver noise at $D$ with $n \sim \mathcal{C N}(0,1)$. The received signal at node $D$ is

$$
y=\sum_{m=1}^{M} g_{m} t_{m}+n,
$$

Define the beamforming vector $\mathbf{w}=\left[w_{1} \ldots w_{M}\right]^{T}$, and the channel vector $\mathbf{h}=\left[\begin{array}{lllll}f_{1} \ldots & f_{M} & g_{1} \ldots g_{M}\end{array}\right]^{T}$. Let

$$
\begin{aligned}
& \mathbf{b} \triangleq\left[\sqrt{\frac{P_{1}}{1+\left|f_{1}\right|^{2} P_{s}}} f_{1} g_{1}, \cdots, \sqrt{\frac{P_{M}}{1+\left|f_{M}\right|^{2} P_{s}}} f_{M} g_{M}\right]^{T} \\
& \mathbf{a} \triangleq\left[\sqrt{\frac{P_{1}}{1+\left|f_{1}\right|^{2} P_{s}}}\left|g_{1}\right|, \cdots, \sqrt{\frac{P_{M}}{1+\left|f_{M}\right|^{2} P_{s}}}\left|g_{M}\right|\right]^{T} \\
& \mathbf{A} \triangleq \operatorname{diag}(\mathbf{a}) .
\end{aligned}
$$

Then, from (1)-(3), the received SNR at node $D$, denoted by $\gamma(\mathbf{h}, \mathbf{w})$, is given by

$$
\gamma(\mathbf{h}, \mathbf{w})=\frac{P_{s}\left|\mathbf{w}^{H} \mathbf{b}\right|^{2}}{1+\|\mathbf{A w}\|^{2}} .
$$

We assume the receiver at destination $D$ has the perfect knowledge of $\mathbf{h}$. With the limited feedback resource, the destination needs to feedback a quantized version of either the channel vector $\mathbf{h}$ (for relays to compute the beamweight $\mathbf{w}$ ), or the beamweight vector $\mathbf{w}$ to all the relays to perform distributed beamforming. Since the dimension of $\mathbf{h}$ is twice as large as $\mathbf{w}$, we directly design the codebook $\mathcal{W}=$ $\left[\mathbf{w}_{1}, \cdots, \mathbf{w}_{K}\right]$ of size $K$ to feedback the best beamweight vector from $\mathcal{W}$. Our codebook design is thus the design of $\mathcal{W}$ such that the capacity of the network is maximized.

Given the codebook $\mathcal{W}$, the maximum capacity between $S$ and $D$ using $\mathrm{AF}$ is

$$
C(\mathbf{h}, \mathcal{W})=\log (1+\tilde{\gamma}(\mathbf{h}, \mathcal{W}))
$$

where $\tilde{\gamma}(\mathbf{h}, \mathcal{W})=\max _{\mathbf{w}_{k} \in \mathcal{W}} \gamma\left(\mathbf{h}, \mathbf{w}_{k}\right)$. To maximize the average end-to-end capacity, the optimal codebook $\mathcal{W}^{o}$ should maximize the average capacity $E_{\mathbf{h}}\{C(\mathbf{h}, \mathcal{W})\}$. Since

$$
E_{\mathbf{h}}\{C(\mathbf{h}, \mathcal{W})\} \leq \log \left(1+E_{\mathbf{h}}\left\{\max _{\mathbf{w}_{k} \in \mathcal{W}} \gamma\left(\mathbf{h}, \mathbf{w}_{k}\right)\right\}\right)
$$

instead of directly using the capacity criterion, we design the codebook using the above capacity upper bound in (6), and we have

$$
\begin{aligned}
& \mathcal{W}^{o}=\underset{\mathcal{W}}{\arg \max } \log \left(1+E_{\mathbf{h}}\left\{\max _{\mathbf{w}_{k} \in \mathcal{W}} \gamma\left(\mathbf{h}, \mathbf{w}_{k}\right)\right\}\right) \\
& =\underset{\mathcal{W}}{\arg \max } E_{\mathbf{h}}\left\{\max _{\mathbf{w}_{k} \in \mathcal{W}} \gamma\left(\mathbf{h}, \mathbf{w}_{k}\right)\right\} \\
& \text { s.t. }\left|w_{m k}\right| \in[0,1] \text {, for } m=1, \cdots, M ; k=1, \cdots, K \text {. }
\end{aligned}
$$

where $w_{m k}$ denotes the $m$-th entry in $\mathbf{w}_{k}$. Thus, the design objective is equivalent to the criterion of maximizing received SNR. Due to shorter relay distance and beamforming gain, we expect the received SNR is typically in the high SNR region. In this region, the bound in (6) is tight. It has been shown that the gap in received SNR by using the approximation in (8) is small in the high SNR region [15]. 


\section{Codebook Design without Power Control}

\section{A. Phase Codebook Design using Grassmannian Line Packing}

Without power control, all the relays always use full power to forward received signal. In this case, the beamweight vector $\mathbf{w}_{k}$ in the codebook $\mathcal{W}$ is only consisted of phase information, and all the power control factors $\alpha_{m k}=1$, for $m=1 \ldots M$ and $k=1 \ldots K$. Thus, the SNR expression in (4) is simplified as

$$
\gamma(\mathbf{h}, \mathbf{w})=\frac{P_{s}\left|\mathbf{w}^{H} \mathbf{b}\right|^{2}}{1+\|\mathbf{a}\|^{2}} .
$$

From (8) and (9), the phase-only codebook $\mathcal{W}^{o}$ design optimization is given as

$\mathcal{W}^{o}=\underset{\mathcal{W}}{\arg \max } E_{\mathbf{h}}[\tilde{\gamma}(\mathbf{h}, \mathcal{W})]=\underset{\mathcal{W}}{\arg \max } E_{\mathbf{h}}\left[\max _{\mathbf{w}_{k} \in \mathcal{W}}\left|\mathbf{b}^{H} \mathbf{w}_{k}\right|^{2}\right]$

$$
\text { s.t. }\left|w_{m k}\right|=1 \text {, for } m=1 \ldots M, k=1 \ldots K
$$

The expression in (10) shows that the distributed relay beamforming design can be viewed as the transmit beamforming design in an equivalent MISO point-to-point system, where $\mathbf{b}$ is the equivalent MISO channel state. In addition, per antenna transmit power constraints are imposed in this equivalent MISO system (i.e., power control factor $\left|w_{m k}\right|=1$, for $m=1, \ldots, M ; k=1, \ldots, K)$. Thus, our phase-only codebook design problem for distributed relay beamforming system is similar to the quantized equal gain transmission (QEGT) codebook design problem [2] in the MISO pointto-point system ${ }^{1}$. Note that, the distribution of the equivalent channel $\mathbf{b}$ in our distributed relay system is different from that of the MISO point-to-point Rayleigh fading system in [2]. Each element in b consists of two concatenated Rayleigh fading channel variables. However, the difference is related to the amplitude component, while the phase component remains uniformly distributed in $[-\pi, \pi]$. Thus, Grassmannian Linear Packing (GLP) criterion [2] can be used to design our phaseonly codebook. Let $\Omega_{M}^{K}=\left\{\mathcal{W}: \forall m, k,\left|\mathbf{w}_{m k}\right|=1\right\}$, then the GLP criterion is given by

$$
\mathcal{W}^{o}=\underset{\mathcal{W} \in \Omega_{M}^{K}}{\arg \max } \delta(\mathcal{W})
$$

where $\delta(\mathcal{W})$ denotes the minimum distance among vector pairs in $\mathcal{W}$, i.e.,

$$
\delta(\mathcal{W})=\min _{1 \leq i<j \leq K} d\left(\mathbf{w}_{i}, \mathbf{w}_{j}\right)
$$

where $d\left(\mathbf{w}_{i}, \mathbf{w}_{j}\right)$ denotes the distance of two complex vectors

$$
d\left(\mathbf{w}_{i}, \mathbf{w}_{j}\right) \triangleq \sqrt{1-\frac{\left|\mathbf{w}_{i}^{H} \mathbf{w}_{j}\right|^{2}}{\left|\mathbf{w}_{i}\right|\left|\mathbf{w}_{j}\right|}},
$$

where $\left|w_{m i}\right|=\left|w_{m j}\right|=1$, for $m=1, \cdots, M$, and $1 \leq i, j \leq$ $K$.

\footnotetext{
${ }^{1}$ Note that it is shown in [2] that for point-to-point systems, the problem of finding quantized beamformers for MISO systems is the same as that for MIMO systems.
}

Although analytical methods [4] or numerical methods [16] can be used to design QEGT Grassmannian codebooks, the codebook comparison experiments in [2] show that random search based designs often produce codebooks with the maximal $\delta(\mathcal{W})$. Thus, we use a random search phase-codebook algorithm (RSPA) to find optimal phase codebook under Grassmannian beamforming criterion (11).

\section{B. Optimal Phase-Only Codebook using Lloyd's Algorithm}

For comparison purpose, we also propose an iterative Lloyd's Algorithm (LA) with a modified distortion measure [5] and a Global Search (GS) [17] method for the optimal codebook design.

To apply LA for codebook design, we define a modified distortion function as

$$
D(\mathcal{W})=E_{\mathbf{h}}\left\{\tilde{\gamma}\left(\mathbf{h}, \mathcal{W}^{o}\right)-\tilde{\gamma}(\mathbf{h}, \mathcal{W})\right\}
$$

where $\tilde{\gamma}\left(\mathbf{h}, \mathcal{W}^{o}\right)$ is the maximum received SNR under the optimal codebook $\mathcal{W}^{o}$. Based on the above distortion function, LA is summarized below.

1) Initialize a codebook $\mathcal{W}^{(0)}=\left[\mathbf{w}_{1}, \cdots, \mathbf{w}_{K}\right]$ of size $K$, with $\left|w_{m k}\right|=1$, for $m=1, \cdots, M ; k=1, \cdots, K$. Set $l=1$.

2) Partition the set $\mathcal{H}$ of possible channel realization vectors into $K$ regions $\mathcal{H}_{k}^{(l)}, k=1 \ldots K$ defined as

$$
\mathcal{H}_{k}^{(l)}=\left\{\mathbf{h}: \gamma\left(\mathbf{h}, \mathbf{w}_{i}^{(l-1)}\right) \leq \gamma\left(\mathbf{h}, \mathbf{w}_{k}^{(l-1)}\right), \forall i \neq k\right\}
$$

3) Generate a new codebook $\mathcal{W}^{(l)}$ with the $k$ th beamforming vector $\mathbf{w}_{k}^{(l)}$ given by

$$
\mathbf{w}_{k}^{(l)}=\underset{\substack{\mathbf{w}:\left|w_{m}\right|=1 \\ m=1, \cdots, M .}}{\arg \max } \quad E_{\mathbf{h} \in \mathcal{H}_{k}^{(l)}}\{\gamma(\mathbf{h}, \mathbf{w})\}
$$

for $k=1, \cdots, K$. For the received $\operatorname{SNR} \gamma(\mathbf{h}, \mathbf{w})$ given in (4), we use the GS method to find the optimal $\mathbf{w}_{k}^{(l)}$ in (16).

4) If $\Delta^{(l)} \triangleq D\left(\mathcal{W}^{(l-1)}\right)-D\left(\mathcal{W}^{(l)}\right)>\epsilon$, where $\epsilon$ is set as the stopping criterion, set $l=l+1$ and go back to step 2. Otherwise, set $\mathcal{W}=\mathcal{W}^{(l)}$ and terminate the iteration procedure.

\section{Codebook Design with Power Control}

In this section, we consider codebook design with power control for the distributed relay beamforming system. The optimal beamforming vector under distributed relay beamforming with per relay power constraints, for a given channel realization, has been obtained in [8]. In the codebook design, we need to jointly quantize the optimal beamforming vector including both phase and power control factors.

\section{A. Optimal Codebook Design with Power Control}

Unlike in the MISO Raleigh fading system, the difficulty in obtaining the optimal codebook for distributed relay beamforing is two-fold. First, as mentioned earlier, due to two-hop relaying structure, each entry in the equivalent MISO channel b consists of two concatenated Rayleigh flat fading channels. 
Thus, the optimal beamweight vector is no longer uniformly distributed in the vector space. Second, due to the individual relay power constraints, each element in a beamforming vector $\mathbf{w}_{k}$ has the amplitude as power control factor with constraint $\left|w_{m k}\right| \leq 1$, for $m=1, \cdots, M$. Both factors above violate the conditions for which the Grassmannian codebook would be optimal, as established in the MIMO point-to-point Rayleigh fading system [2], [3], or random vector quantization (RVQ) codebook [1].

Instead, we design the optimal codebook using the iterative LA method and GS method. The detailed steps are similar to those listed for generating phase-only codebook in Section III$\mathrm{B}$, except that, in using GS to find the optimal $\mathbf{w}_{k}^{(l)}$ in (16), the constraint on $\mathbf{w}$ changes to $\left|w_{m}\right| \leq 1$, for $m=1, \cdots, M$. Details are omitted to avoid repetition.

\section{B. Suboptimal Codebook Design with Power Control}

For the optimal codebook design using the above combined LA and GS method, the power control factors and phase component of beamforming weight are quantized jointly. The drawback of this method is the high computational complexity, due to the complexity involved in the GS method to find the optimal beamforming weight vector in (16). In practice, to generate codebook online, we propose a low-complexity suboptimal codebook design in which phase sub-codebook $\mathcal{W}_{\theta}$ and power control sub-codebook $\mathcal{W}_{\alpha}$ are designed separately.

1) Phase sub-codebook: For the phase sub-codebook $\mathcal{W}_{\theta}$, we again use the GLP criterion (11), and generate the phase sub-codebook.

2) Power control sub-codebook: We design the power control sub-codebook $\mathcal{W}_{\alpha}$, assuming the perfect phase information. In this case, it can be seen from (9) that the optimal phase component for each beamweight $w_{m}=\alpha_{m} e^{j \theta_{m}}$ is given as $\theta_{m}^{o}=\angle f_{m}+\angle g_{m}$, for $m=1, \cdots, M$. In other words, the optimal phase is to cancel out the phase of the aggregated channel from the two hops, so that signals from different relays will be coherently added. Under this assumption, the received SNR is given as

$$
\gamma(\mathbf{h}, \mathbf{w})=\gamma\left(\mathbf{h}, \boldsymbol{\alpha} \mid \boldsymbol{\theta}^{o}\right)=\frac{P_{s}\left|\boldsymbol{\alpha}^{T} \tilde{\mathbf{b}}\right|^{2}}{1+\|\mathbf{A} \boldsymbol{\alpha}\|^{2}}
$$

where

$$
\tilde{\mathbf{b}} \triangleq\left[\sqrt{\frac{P_{1}}{1+\left|f_{1}\right|^{2} P_{s}}}\left|f_{1} g_{1}\right|, \cdots, \sqrt{\frac{P_{M}}{1+\left|f_{M}\right|^{2} P_{s}}}\left|f_{M} g_{M}\right|\right]^{T}
$$$$
\boldsymbol{\alpha} \triangleq\left[\alpha_{1}, \cdots, \alpha_{M}\right]^{T}, \quad \boldsymbol{\theta}^{o}=\left[\theta_{1}^{o}, \cdots, \theta_{M}^{o}\right]^{T} .
$$

Let $\tilde{\gamma}\left(\mathbf{h}, \mathcal{W}_{\alpha}\right) \triangleq \max _{\alpha \in \mathcal{W}_{\alpha}} \gamma\left(\mathbf{h}, \boldsymbol{\alpha} \mid \boldsymbol{\theta}^{o}\right)$. Our power control sub-codebook design is given by

$$
\mathcal{W}_{\alpha}^{o}=\underset{\mathcal{W}_{\alpha}}{\arg \max } E_{\mathbf{h}}\left[\tilde{\gamma}\left(\mathbf{h}, \mathcal{W}_{\alpha}\right)\right]
$$

We again use LA with a modified distortion function to solve (18). The modified distortion function is given by

$$
D\left(\mathcal{W}_{\alpha}\right)=E_{\mathbf{h}}\left[\tilde{\gamma}\left(\mathbf{h}, \mathcal{W}_{\alpha}^{o}\right)-\tilde{\gamma}\left(\mathbf{h}, \mathcal{W}_{\alpha}\right)\right]
$$

The LA method for obtaining $\mathcal{W}_{\alpha}^{o}$ follows the same four-step procedure described in Section III-B. The only modification is (16) in step 3 , where the new beamforming weight vector $\mathbf{w}_{k}^{(l)}$ is obtained for the partitioned channel region $\mathcal{H}_{k}^{(l)}$. It is now given by

$$
\begin{aligned}
& \boldsymbol{\alpha}_{k}^{(l)}= \underset{\substack{\boldsymbol{\alpha}: \boldsymbol{\alpha}_{m} \leq 1 \\
m=1, \cdots, M .}}{\arg \max } \quad E_{\mathbf{h} \in \mathcal{H}_{k}^{(l)}}\left[\gamma\left(\mathbf{h}, \boldsymbol{\alpha} \mid \boldsymbol{\theta}^{o}\right)\right] \\
&=\underset{\substack{\boldsymbol{\alpha}: \boldsymbol{\alpha}_{m} \leq 1 \\
m=1, \cdots, M .}}{\arg \max } \quad E_{\mathbf{h} \in \mathcal{H}_{k}^{(l)}}\left[\frac{P_{s}\left|\boldsymbol{\alpha}^{T} \tilde{\mathbf{b}}\right|^{2}}{1+\|\mathbf{A} \boldsymbol{\alpha}\|^{2}}\right]
\end{aligned}
$$

Note that the above optimization is the main step associated with high computation complexity. In particular, the expectation in the objective function makes the optimization problem difficult to solve, and either exhaustive search or other numerical search methods, such as GS, need to be used to find $\boldsymbol{\alpha}_{k}^{(l)}$. In our suboptimal codebook design, instead of using the numerical method to search for the optimal $\boldsymbol{\alpha}_{k}^{(l)}$, we propose the following method to obtain $\boldsymbol{\alpha}_{k}^{(l)}$. The expression in (20) is approximated as

$$
\begin{aligned}
E_{\mathbf{h} \in \mathcal{H}_{k}^{(l)}}\left[\frac{P_{s}\left|\boldsymbol{\alpha}^{T} \tilde{\mathbf{b}}\right|^{2}}{1+\|\mathbf{A} \boldsymbol{\alpha}\|^{2}}\right] & \approx \frac{P_{s} E_{\mathbf{h} \in \mathcal{H}_{k}^{(l)}}\left[\left|\boldsymbol{\alpha}^{T} \tilde{\mathbf{b}}\right|^{2}\right]}{1+E_{\mathbf{h} \in \mathcal{H}_{k}^{(l)}}\left[\|\mathbf{A} \boldsymbol{\alpha}\|^{2}\right]} \\
& =\frac{P_{s} \boldsymbol{\alpha}^{T} E_{\mathbf{h} \in \mathcal{H}_{k}^{(l)}}\left[\tilde{\mathbf{b}} \tilde{\mathbf{b}}^{T}\right] \boldsymbol{\alpha}}{1+\boldsymbol{\alpha}^{T} E_{\mathbf{h} \in \mathcal{H}_{k}^{(l)}}\left[\mathbf{A} \mathbf{A}^{T}\right] \boldsymbol{\alpha}} \\
& =\frac{P_{s} \boldsymbol{\alpha}^{T} \mathbf{B}_{k} \boldsymbol{\alpha}}{1+\boldsymbol{\alpha}^{T} \mathbf{Q}_{k} \boldsymbol{\alpha}}
\end{aligned}
$$

where $\mathbf{B}_{k} \triangleq E_{\mathbf{h} \in \mathcal{H}_{k}^{(l)}}\left[\tilde{\mathbf{b}} \tilde{\mathbf{b}}^{T}\right]$, and $\mathbf{Q}_{k} \triangleq E_{\mathbf{h} \in \mathcal{H}_{k}^{(l)}}\left[\mathbf{A A}^{T}\right]$ where $\mathbf{Q}_{k}$ is diagonal. Note that, for a given $\mathbf{h}, \tilde{\mathbf{b}} \tilde{\mathbf{b}}^{T}$ has rank one structure. However, after averaging over $\mathcal{H}_{k}^{(l)}$, $\mathbf{B}_{k}$ can be a full rank matrix. In order to preserve the rank-one structure of the original SNR expression, we take the eigenvalue decomposition of $\mathbf{B}$ as $\mathbf{B}_{k}=\mathbf{U}_{k} \boldsymbol{\Lambda}_{k} \mathbf{U}_{k}{ }^{T}$, where $\boldsymbol{\Lambda}_{k}=\operatorname{diag}\left[\lambda_{1 k}, \cdots, \lambda_{M k}\right]$, and $\lambda_{1 k}$ is the largest eigenvalue of $\mathbf{B}_{k}$. The corresponding eigenvector matrix is $\mathbf{U}_{k}=\left[\mathbf{u}_{1 k}, \cdots, \mathbf{u}_{M k}\right]$, where $\mathbf{u}_{m k}$ is the eigenvector of $\mathbf{B}_{k}$ corresponding to $\lambda_{m k}, m=1, \cdots, M$. Then, we approximate $\mathbf{B}_{k}$ by

$$
\mathbf{B}_{k} \approx \lambda_{1 k} \mathbf{u}_{1 k} \mathbf{u}_{1 k}^{T}
$$

Let $\overline{\mathbf{b}}_{k} \triangleq \sqrt{\lambda_{1 k}}\left|\mathbf{u}_{1 k}\right|$, where $\left|\mathbf{u}_{1 k}\right|$ denotes the element-wise absolute value of $\mathbf{u}_{1 k}{ }^{2}$. Let $\overline{\mathbf{A}}_{k} \triangleq \mathbf{Q}_{k}^{1 / 2}$. Since $\mathbf{Q}_{k}$ is diagonal, $\overline{\mathbf{A}}_{k}$ is also diagonal. Then, (22) becomes

$$
E_{\mathbf{h} \in \mathcal{H}_{k}^{(l)}}\left[\frac{P_{s}\left|\boldsymbol{\alpha}^{T} \tilde{\mathbf{b}}\right|^{2}}{1+\|\mathbf{A} \boldsymbol{\alpha}\|^{2}}\right] \approx \frac{P_{s}\left|\boldsymbol{\alpha}^{T} \overline{\mathbf{b}}_{k}\right|^{2}}{1+\left\|\overline{\mathbf{A}}_{k} \boldsymbol{\alpha}\right\|^{2}}
$$

\footnotetext{
${ }^{2}$ Note that entries in $\mathbf{u}_{1 k}$ may be negative. But, the negative phase will be compensated by the corresponding beamforming weights which cancel out the phase.
} 
The optimization problem in (20) now is changed to

$$
\boldsymbol{\alpha}_{k}^{(l)}=\underset{\substack{\boldsymbol{\alpha}: \alpha_{m} \leq 1 \\ m=1, \cdots, M .}}{\arg \max } \frac{P_{s}\left|\boldsymbol{\alpha}^{T} \overline{\mathbf{b}}_{k}\right|^{2}}{1+\left\|\overline{\mathbf{A}}_{k} \boldsymbol{\alpha}\right\|^{2}}
$$

The above objective function can be viewed as the received SNR of a distributed relay beamforming system with an equivalent channel over each hop defined as

$$
\begin{aligned}
\bar{f}_{m k} & \triangleq \bar{b}_{m k} / \bar{a}_{m k} \\
\bar{g}_{m k} & \triangleq \bar{a}_{m k} \sqrt{\frac{1+\left|\bar{f}_{m k}\right|^{2} P_{0}}{P_{R}}} \\
\overline{\mathbf{h}}_{k} & \triangleq\left[\bar{f}_{1 k}, \cdots, \bar{f}_{M k}, \bar{g}_{1 k}, \cdots, \bar{g}_{M k}\right]^{T}
\end{aligned}
$$

where $\bar{b}_{m k}$ and $\bar{a}_{m k}$ is the $m$ th element in $\overline{\mathbf{b}}_{k}$ and the $m$ th diagonal element in $\overline{\mathbf{A}}_{k}$, respectively. Essentially, through approximation, we obtain an equivalent channel $\overline{\mathbf{h}}_{k}$ in (26) representing the $k$ th channel partition region $\mathcal{H}_{k}^{(l)}$, and we convert the optimization problem in (20) to that of finding the optimal power control vector $\boldsymbol{\alpha}_{k}^{(l)}$ for the equivalent distributed relay beamforming system associated to $\overline{\mathbf{h}}_{k}$, as shown in (25). The solution to (25) is readily obtained by [8, Theorem 1].

3) Combining phase and power control sub-codebooks: With the phase sub-codebook $\mathcal{W}_{\theta}$ and power control subcodebook $\mathcal{W}_{\alpha}$ generated separately, we now combine the two sub-codebooks into one codebook $\mathcal{W}$. There are two approaches to combine the two sub-codebooks:

1) Combine each power control vector $\boldsymbol{\alpha}_{i} \in \mathcal{W}_{\alpha}$ with each phase vector $\boldsymbol{\theta}_{j} \in \mathcal{W}_{\theta}$ to form the amplitude and phase part of a beamforming weight vector in $\mathcal{W}$. The resulting size of $\mathcal{W}$ is $K=K_{\alpha} \times K_{\theta}$. For a given codebook size $K$, this approach requires us to first determine the size $K_{\theta}$ of phase sub-codebook and the size $K_{\alpha}$ of the power control sub-codebook, such that $K=K_{\alpha} \times K_{\theta}$.

2) Generate $\mathcal{W}_{\alpha}$ and $\mathcal{W}_{\theta}$ with size $K_{\alpha}=K_{\theta}=K$, then randomly choose $\boldsymbol{\alpha}_{i} \in \mathcal{W}_{\alpha}$ and $\boldsymbol{\theta}_{j} \in \mathcal{W}_{\theta}$ to combine as a new beamforming weight vector for the codebook $\mathcal{W}$. Each vector in $\mathcal{W}_{\alpha}$ or $\mathcal{W}_{\theta}$ can only be used once in forming $\mathcal{W}$.

Comparing the two approaches, the first approach requires smaller sub-codebook size $K_{\alpha}$ and $K_{\theta}$ for $\mathcal{W}_{\alpha}$ and $\mathcal{W}_{\theta}$, respectively, leading to more quantization loss. In addition, different choices for $K_{\alpha}$ and $K_{\theta}$ that lead to the same $K=$ $K_{\alpha} \times K_{\theta}$ will result in different performance, and therefore further optimization in the combination of $K_{\alpha}$ and $K_{\theta}$ needs to be considered. The second approach overcomes both issues mentioned above, at the price of allowing each power control vector in $\mathcal{W}_{\alpha}$ to be combined with only one of the phase vectors in $\mathcal{W}_{\theta}$, which results in quantization error due to limitation in combining. The simulations show that when the codebook size is small, the second approach can provide better performance with smaller average quantization error.

\section{Simulation Results}

To compare the performance of different codebooks, we consider a two-hop distributed relay network with $M=4$ relays. We assume the first hop and second hop channel $f_{m}$ and $g_{m}$ are i.i.d Rayleigh flat fading. Noise power is set to $1 \mathrm{~W}$. We define transmit SNR as the transmit power over noise power.

Fig. 1 compares the average received SNR performance of phase-only codebooks using RSPA under GLP criterion, and the combined LA and GS method, for codebook size $K=$ 16,64 , respectively. The Rayleigh fading channels $f_{m}$ and $g_{m}$ are generated with distribution $\mathcal{C N}(0,1)$ for $m=1, \ldots M$. We see that the performance under the two methods is nearly identical. The codebook using RSPA can be generated offline, and independent of any specific channel realization set. Thus, phase-only codebook by RSPA under GLP criterion is preferred.

To see the performance of codebooks with power control, we plot the average received SNR at different relay locations in Fig. 2. We set $P_{o}=P_{r}=20 \mathrm{dBW}$. The codebooks are generated using the optimal LA with GS method, and the suboptimal method, for $K=16$ and $K=64$, respectively. The suboptimal codebook refers to the approaches in Section IV-B with $K_{\alpha}=K_{\theta}=4$ for $K=16$, and $K_{\alpha}=K_{\theta}=8$ for $K=$ 64 . We set the distance between source and destination is 1 . We assume all relays located at the same location, and denote $d 1$ as the distance between relays to the source node. The channel variances for $f_{m}$ and $g_{m}$ are determined based on the pathloss model, i.e., $f_{m} \sim \mathcal{C N}\left(0, d_{1}^{-2}\right), g_{m} \mathcal{C N}\left(0,\left(1-d_{1}\right)^{-2}\right)$, for $m=1, \cdots, M$.. We see that the codebook performance are close to parallel to the case of with perfect CSI. The optimal LA with GS codebook of size $K=64$ is $0.5 \sim 1.5 d B$ lower than the perfect CSI case and approximately $1 d B$ higher than the optimal LA with GS codebook of size $K=16$. The suboptimal codebooks are approximately $0.5 d B$ lower than the optimal LA with GS codebook with corresponding codebook size. The simulation shows that the codebook quantization loss is not sensitive the relay locations.

Finally, to see the effect of relay location on power control gain, we plot the SNR gain of codebook with power control over phase-only codebook at different relay locations in Fig. 3. The SNR gain of optimal power control over no power control (full power) under the perfect CSI is also plotted. For codebook with power control, we use the combined LA and GS method. As we can see, the power control gain increases as relays move from source $S$ to destination $D$. When relays are close to the source $\mathrm{S}$, the power control gain is negligible, and there could be even a loss due to quantization. This shows that the selection of the type of codebooks, i.e., with or without power control, is location dependent.

\section{CONCLuSiOnS}

In this paper, we focused on the problem of codebook design for distributed relay beamforming under individual relay power constraint. Our performance metric was the received SNR. We separately studied two types of codebook design: phase-only codebook, and codebook with power control where relay power and phase need to be jointly considered. For phase-only codebook, we adopt Grassmannian line packing 


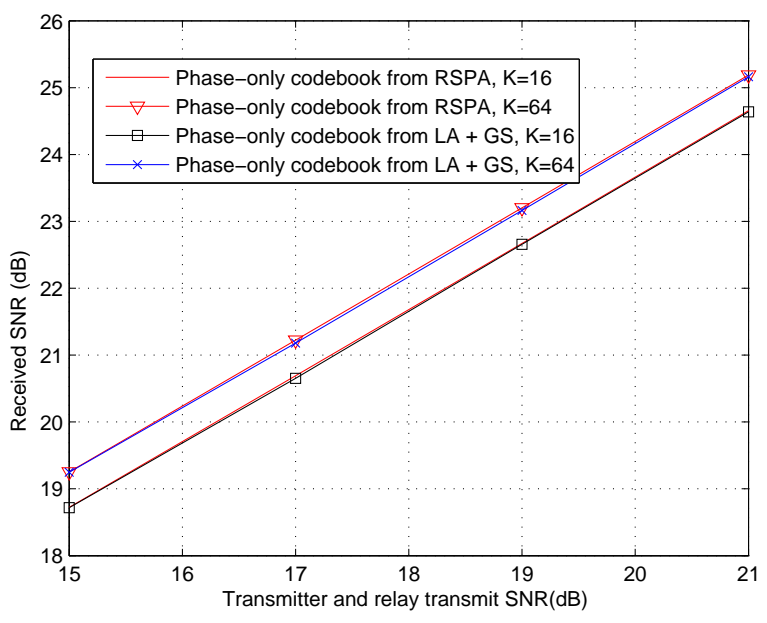

Fig. 1. Received SNR vs. transmit SNR under phase-only codebooks $\left(P_{o}=\right.$ $P_{r}, M=4$.).

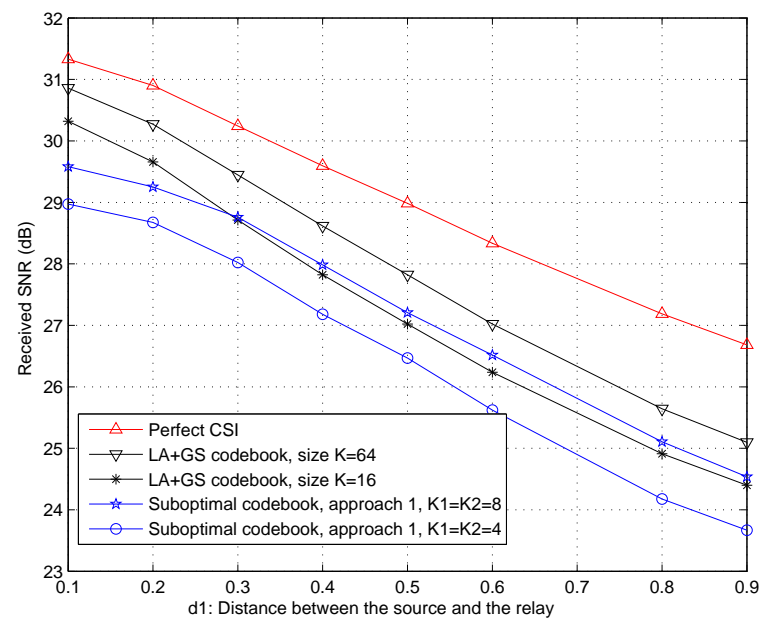

Fig. 2. Received SNR vs. relay locations under codebooks with power control $\left(P_{o}=P_{r}=20 \mathrm{dBW}, M=4\right)$

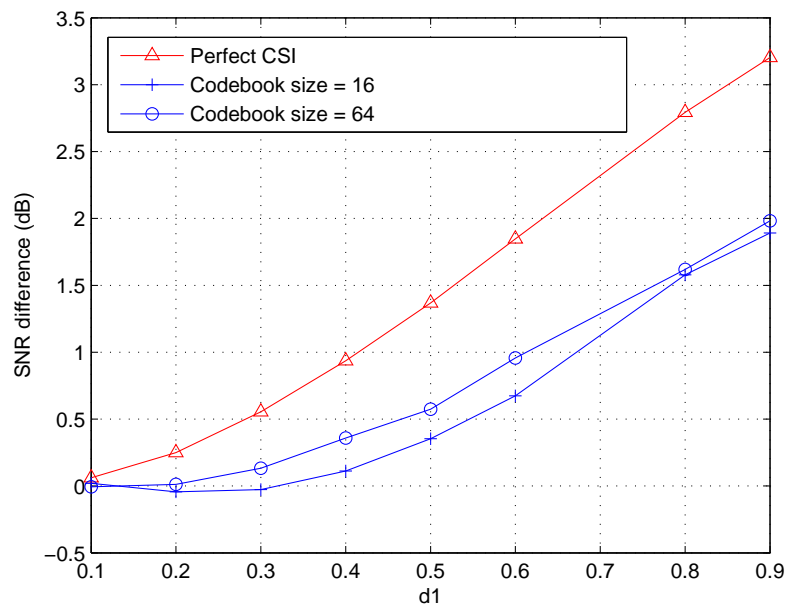

Fig. 3. Performance gain of power control vs. relay locations $\left(P_{o}=P_{r}=\right.$ $20 \mathrm{dBW}, M=4)$ criterion, which can generate codebooks offline. Simulation showed only negligible performance loss as compared to that under the generalized Lloyd Algorithm for optimal codebook design. For codebook with power control, besides the generalized Lloyd Algorithm for the optimal codebook design, we proposed a low-complexity suboptimal method, which can directly use the optimal power control solution for perfect CSI under the individual power constraint. We observed from the simulations that, the gain of codebook with power control over phase-only codebook depends on relay locations, and the codebook with power control is beneficial when relays are closer to destination, instead of the source.

\section{REFERENCES}

[1] C. K. Au-Yeung and D. Love, "On the performance of random vector quantization limited feedback beamforming in a miso system," IEEE Trans. Wireless Commun., vol. 6, pp. 458 -462, Feb. 2007.

[2] D. Love, R. Heath, and T. Strohmer, "Grassmannian beamforming for multiple-input multiple-output wireless systems," IEEE Trans. Inform. Theory, vol. 49, pp. 2735 - 2747, Oct. 2003.

[3] K. Mukkavilli, A. Sabharwal, E. Erkip, and B. Aazhang, "On beamforming with finite rate feedback in multiple-antenna systems," IEEE Trans. Inform. Theory, vol. 49, pp. 2562 - 2579, Oct. 2003.

[4] T. Strohmer and R. W. H. Jr., "Grassmannian frames with applications to coding and communications," Appl. Comput. Harm. Anal., vol. 14, pp. 257-275, 2002.

[5] Y. Linde, A. Buzo, and R. Gray, "An algorithm for vector quantizer design," IEEE Trans. Commun., vol. 28, pp. 84 - 95, Jan. 1980.

[6] P. Larsson, "Large-scale cooperative relaying network with optimal combining under aggregate relay power constraint," in Proc. of Future Telecomm. Conf., Jun. 2003, pp. 160-170.

[7] I. Maric and R. Yates, "Bandwidth and power allocation for cooperative strategies in gaussian relay networks," IEEE Trans. Inform. Theory, vol. 56, pp. $1880-1889$, Apr. 2010.

[8] Y. Jing and H. Jafarkhani, "Network beamforming using relays with perfect channel information," IEEE Trans. Inform. Theory, vol. 55, pp. $2499-2517$, Jun. 2009.

[9] M. Dong and S. Shahbazpanahi, "Optimal spectrum sharing and power allocation for ofdm-based two-way relaying," in Proc. IEEE Int. Conf. Acoustics, Speech, and Signal Processing (ICASSP), Mar. 2010, pp. 3310 -3313 .

[10] S. Shahbazpanahi and M. Dong, "Achievable rate region and sumrate maximization for network beamforming for bi-directional relay networks," in Proc. IEEE Int. Conf. Acoustics, Speech, and Signal Processing (ICASSP), Mar. 2010, pp. $2510-2513$

[11] N. Ahmed, M. Khojastepour, A. Sabharwal, and B. Aazhang, "Outage minimization with limited feedback for the fading relay channel," IEEE Trans. Commun., vol. 54, pp. 659 - 669, Apr. 2006.

[12] Y. Zhao, R. Adve, and T. Lim, "Beamforming with limited feedback in amplify-and-forward cooperative networks - [transactions letters]," IEEE Trans. Wireless Commun., vol. 7, pp. 5145 -5149, Dec. 2008

[13] M. Hajiaghayi, M. Dong, and B. Liang, "Using limited feedback in power allocation design for a two-hop relay OFDM system," in Proc. IEEE Int. Conf. Communications (ICC), vol. 2, Jun. 2009, pp. 11671171.

[14] B. Khoshnevis and W. Yu, "Joint power control and beamforming codebook design for miso channels with limited feedback," in Proc.IEEE Global Telecommn. Conf. (GLOBECOM), Dec. 2009, pp. 1 -6.

[15] V. Lau and T. Wu, "Optimal transmission and limited feedback design for OFDM/MIMO systems in frequency selective block fading channels," IEEE Trans. Wireless Commun., vol. 6, pp. 1569 -1573, May 2007.

[16] D. Agrawal, T. Richardson, and R. Urbanke, "Multiple-antenna signal constellations for fading channels," IEEE Trans. Inform. Theory, vol. 47, pp. $2618-2626$, Sep. 2001

[17] E. Talbi, Metaheuristics-from design to implementation. Hoboken, New Jersey: John Wiley \& Sons, Inc., 2009. 